\title{
The Cutoff Values of HOMA-IR in Childhood and Trends Changing with Sexual Maturation Stage
}

\section{Oya YÜCEL}

Başkent University, Istanbul Hospital, Turkey

\section{Alev BAKIR}

Haliç University, Istanbul, Turkey

Homeostasis model assessment (HOMA) was found to be reliable in diagnosis of IR in obese children. The main problem is the lack of ranges for insulin and HOMA-IR linked to gender, age, sexual maturation stage and obesity. The present study aimed to determine trends of variation in HOMA-IR taking pubertal stages into account and to compare children with obesity and those without obesity.

Methods: The cross-sectional prospective study. Obesity; BMI $\geq 95$ th percentile for age and sex (CDC criteria). The studied variables were age, gender, lipid profile, thyroid function tests, fasting serum glucose and insulin. HOMA score (fasting serum insulin $(\mu \mathrm{u} / \mathrm{ml}) \mathrm{X} \mathrm{FGlu} \mathrm{(mg/dl)} / 405)$. Children were divided into three groups: pre-pubertal (Tanner's I), mid-pubertal (Tanner's II-IV) and post-pubertal (Tanner’s V).

Table 1: The mean values of HOMA-IR based on gender/obesity

\begin{tabular}{|c|c|c|c|c|c|c|c|c|c|c|}
\hline Ages & $\mathrm{n}$ & $\begin{array}{c}\text { Total } \\
\operatorname{an} \pm \mathrm{SD} / \text { Median }\end{array}$ & & $\begin{array}{c}\text { Girls } \\
\text { ean } \pm \text { SD/Median }\end{array}$ & $\mathrm{n}$ & $\begin{array}{c}\text { Boys } \\
\text { and } \pm \text { SD/Median }\end{array}$ & $\mathrm{n}$ & $\begin{array}{c}\text { Non-obese } \\
\text { ean } \pm \text { SD/Median }\end{array}$ & $\mathrm{n}$ & $\begin{array}{c}\text { Obese } \\
\text { Mean } \pm \text { SD/Median }\end{array}$ \\
\hline $7 y$ & 16 & $2.32 \pm 1.00 / 2.08$ & 6 & $2.34 \pm 0.59 / 2.43$ & 10 & $2.30 \pm 1.21 / 1.93$ & 1 & $1.56 \pm-/-$ & 15 & $2.37 \pm 1.01 / 2.13$ \\
\hline $8 y$ & 9 & $1.63 \pm 0.95 / 1.53$ & 6 & $1.31 \pm 0.43 / 1.39$ & 3 & $2.28 \pm 1.47 / 1.74$ & 4 & $1.25 \pm 0.52 / 1.34$ & 5 & $1.94 \pm 1.15 / 1.60$ \\
\hline $9 y$ & 25 & $2.32 \pm 1.19 / 2.31$ & 16 & $2.35 \pm 1.30 / 2.18$ & 9 & $2.26 \pm 1.04 / 2.84$ & 6 & $1.21 \pm 0.42 / 1.10$ & 19 & $2.67 \pm 1.14 / 2.78$ \\
\hline $10 \mathrm{y}$ & 19 & $2.62 \pm 1.15 / 2.29$ & 12 & $2.77 \pm 1.26 / 2$ & 7 & $2.37 \pm 0.96 /$ & 9 & $1.99 \pm 0.90 / 1.79$ & 10 & $3.19 \pm 1$ \\
\hline $11 y$ & 27 & $2.78 \pm 1.63 / 2.69$ & 8 & $2.63 \pm 1.17 / 2.76$ & 19 & $2.84 \pm 1.81 /$ & 11 & $1.88 \pm 0.8$ & 16 & $6 / 3.12$ \\
\hline $12 \mathrm{y}$ & 28 & $3.42 \pm 2.31 / 2.86$ & 14 & $4.36 \pm 2.81 / 3.51$ & 14 & $2.48 \pm 1.15 / 2.21$ & 13 & $2.26 \pm 0.86 / 2.00$ & 15 & $4.43 \pm 2.71 / 3.53$ \\
\hline $13 y$ & 29 & $3.74 \pm 2.48 / 3.48$ & 10 & $4.30 \pm 3.69 / 3.34$ & 19 & $3.44 \pm 1.57 .3 .48$ & 18 & $3.02 \pm 1.48 / 2.80$ & 11 & $4.91 \pm 3.32 / 4.11$ \\
\hline $14 y$ & 20 & $2.80 \pm 1.20 / 2.56$ & 11 & $2.74 \pm 1.25 / 2.26$ & 9 & $2.87 \pm 1.22 / 2.78$ & 10 & $2.51 \pm 1.21 / 2.49$ & 10 & $3.08 \pm 1.19 / 2.98$ \\
\hline $15 \mathrm{y}$ & 12 & $3.19 \pm 1.81 / 2.55$ & 7 & $3.16 \pm 2.21 / 2.46$ & 5 & $3.24 \pm 1.29 / 2.65$ & 4 & $2.08 \pm 0.85 / 2.12$ & 8 & $3.75 \pm 1.94 / 3.26$ \\
\hline $16 y$ & 16 & $3.52 \pm 2.10 / 3.15$ & 8 & $2.98 \pm 1.34 / 2.82$ & 8 & $4.06 \pm 2.63 / 3.31$ & 8 & $2.44 \pm 1.09 / 2.21$ & 8 & $4.60 \pm 2.36 / 4.17$ \\
\hline $17 y$ & 6 & $2.61 \pm 1.99 / 1.70$ & 2 & $2.40 \pm 2.37 / 2.40$ & 4 & $2.72 \pm 2.17 / 1.70$ & 4 & $2.05 \pm 1.43 / 1.70$ & 2 & $3.73 \pm 3.16 / 3.73$ \\
\hline Total & 207 & $2.92 \pm 1.82 / 2.53$ & 100 & $2.99 \pm 2.05 / 2.43$ & 107 & $2.86 \pm 1.58 / 2.59$ & 88 & $2.24 \pm 1.16 / 1.92$ & 119 & $3.43 \pm 2.04 / 2.93$ \\
\hline
\end{tabular}

Table 2: The cutoff, mean and median values of HOMA-IR to predict IR

\begin{tabular}{|c|c|c|c|c|c|c|c|c|}
\hline & Mean \pm SD & Median & Min/Max & cut-off & $\mathrm{SN}(\%)$ & $\mathrm{SP}(\%)$ & AUC & $\mathrm{p}$ \\
\hline Total & $2.92 \pm 1.82$ & 2.53 & $0.50 / 13.61$ & 1.998 & 77 & 57 & 0.701 & $<0.001 \mathrm{a}^{*}$ \\
\hline Girls & $2.99 \pm 2.05$ & 2.43 & $0.50 / 13.61$ & 1.987 & 79 & 56 & 0.701 & $0.001 \mathrm{a}^{*}$ \\
\hline Boys & $2.86 \pm 1.58$ & 2.59 & $0.84 / 9.34$ & 3.063 & 50 & 84 & 0.704 & $<0.001 \mathrm{a}^{*}$ \\
\hline \multicolumn{9}{|c|}{ Puberty } \\
\hline Pre- & $2.52 \pm 1.32$ & 2.31 & $0.56 / 7.99$ & 1.83 & 74 & 73 & 0.732 & $0.001 \mathrm{a}^{*}$ \\
\hline Mid- & $2.87 \pm 1.43$ & 2.72 & $0.50 / 7.68$ & 3.21 & 70 & 79 & 0.756 & $0.002 \mathrm{a}^{*}$ \\
\hline Post- & $3.45 \pm 2.40$ & 2.86 & $0.73 / 13.61$ & 3.11 & 65 & 78 & 0.764 & $<0.001 \mathrm{a}^{*}$ \\
\hline \multicolumn{9}{|c|}{$p: 0.042 c^{*}($ Pre-Mid py:0.097; Pre-Post py:0.019*; Mid-Post py:0.385) } \\
\hline
\end{tabular}

SD: Standart Deviation, SN: Sensitivity, SP: Specificity, AUC: Area Under Curve. a.ROC Analysis, b.Mann-Whitney Test, c.Kruskal-Wallis Test, * significant p<0.05 or py Bonferroni Correction

\section{Discussion}

The cut-off values of HOMA-IR need to be defined in the pediatric population to diagnose IR. There are a few studies evaluating cut-off values of HOMA-IR in childhood. Individual cutoff points of HOMA-IR based on Youden's index using the ROC curve were defined in the current study. Trends changing with age and the impact of puberty were also evaluated. The cutoff points of HOMA-IR vary from pre-pubertal to post-pubertal stages without no statistically significant difference between sexes. HOMA-IR values have peaked at 12 years of age.

The cutoff values of HOMA-IR for diagnosis of IR were derived from OGTT in some studies with obese children. ROC curves were used to determine the cutoff points of HOMA-IR in these studies. Two studies from Turkey were proposed cutoff points of HOMA-IR $\geq 2.7$ for both sexes (1) and HOMA-IR $>2.2$ for girls and $>2.7$ for boys in pre-pubertal stages (2). Another study demonstrated that the cutoff point was 3.16 in obese adolescents. The cutoff point of 2.5 is valid for adults, but not for adolescents (3).

In pre-pubertal normal weight Italian children, HOMA-IR value was reported of 2.0 as a cutoff point (4).

In the current study, suggested HOMA-IR values were $>1.83$ in pre-pubertal children and $>3.11$ in $\mathrm{mid} /$ post-pubertal stages.

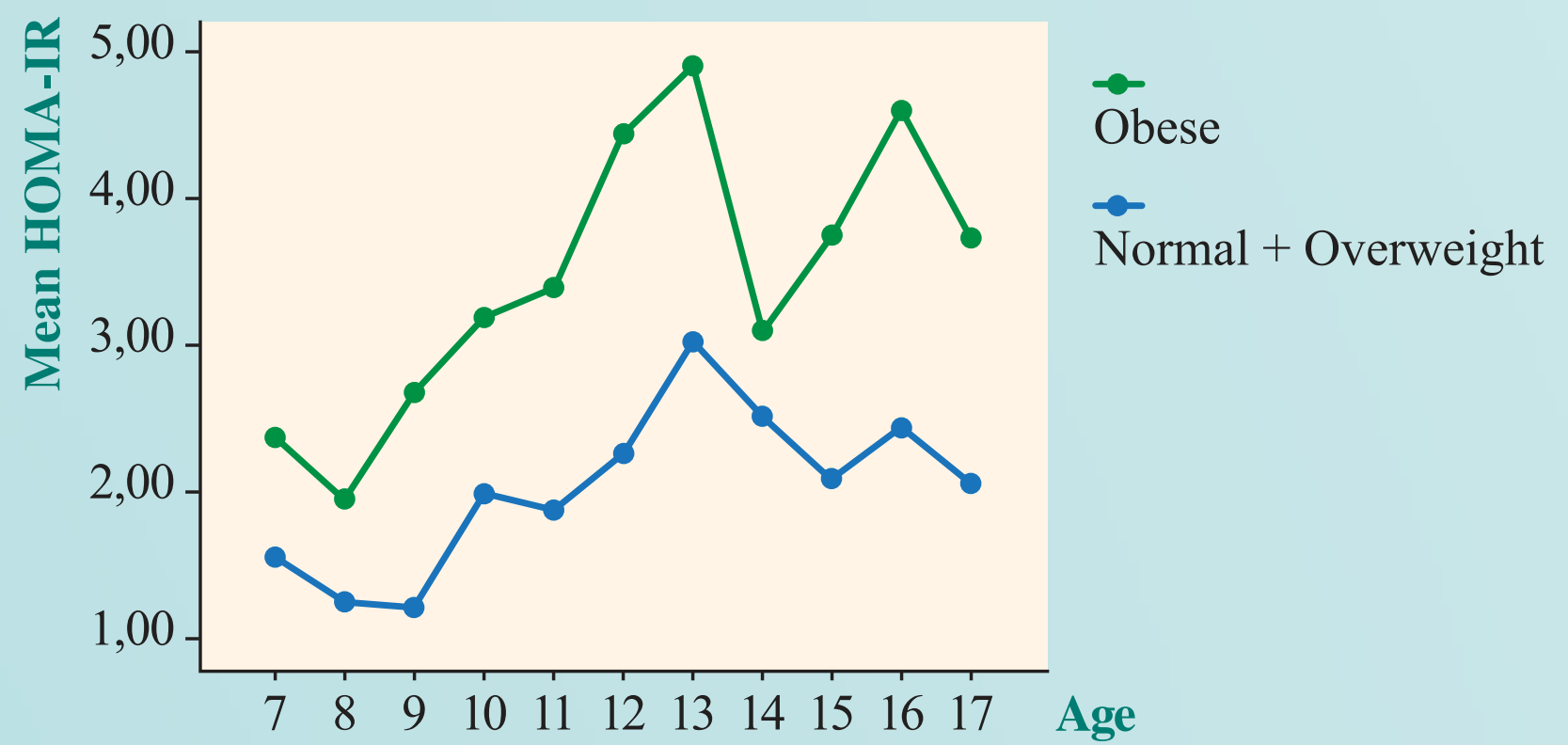

Results:

207 children aged 7 to 17 years (119 obese, 57\%; 88 non-obese) There was no statistical difference in terms of gender. Obese group had higher HOMA-IR values than non-obese group ; (3.43 \pm 2.04 vs $2.24 \pm 1.16$, respectively) $(\mathrm{p}<0.001)$ (Table 3$)$.

The cutoff values; 2.02 in Tanner's I-II-III; 3.11 in Tanner's IV-V

The cutoff values; 1.83 in the pre-pubertal; 3.21 in the mid-pubertal; 3.11 in the post-pubertal group. 